\title{
Prevalence of tick-borne encephalitis virus antibodies in dogs from Denmark
}

\author{
Katherine ES Lindhe*1, Danny S Meldgaard ${ }^{2}$, Per M Jensen ${ }^{3}$, \\ Geoffrey A Houser ${ }^{4}$ and Mette Berendt ${ }^{4}$
}

\author{
Address: 'Vangede Small Animal Veterinary Clinic, Plantevej 2, DK-2870 Dyssegård., Denmark, ${ }^{2}$ Buddinge Small Animal Veterinary Clinic, \\ Buddinge Hovedgade 222, DK-2880 Bagsværd, Denmark, ${ }^{3}$ Department of Agriculture and Ecology, Faculty of Life Sciences, University of \\ Copenhagen, Thorvaldsensvej 40, opg. 2, DK-1871 Frederiksberg C., Denmark and ${ }^{4}$ Department of Small Animal Clinical Sciences, Faculty of Life \\ Sciences, University of Copenhagen, Dyrlægevej 16, DK-1870 Frederiksberg C., Denmark \\ Email: Katherine ES Lindhe* - stipp@dsr.life.ku.dk; Danny S Meldgaard - dsm@dsr.life.ku.dk; Per M Jensen - pmj@life.ku.dk; \\ Geoffrey A Houser - geh@life.ku.dk; Mette Berendt - mbe@life.ku.dk \\ * Corresponding author
}

Published: 29 December 2009

Acta Veterinaria Scandinavica 2009, 5 I:56 doi:10.1 186/I75I-0147-5I-56

This article is available from: http://www.actavetscand.com/content/5I/I/56

(C) 2009 Lindhe et al; licensee BioMed Central Ltd.

This is an Open Access article distributed under the terms of the Creative Commons Attribution License (http://creativecommons.org/licenses/by/2.0), which permits unrestricted use, distribution, and reproduction in any medium, provided the original work is properly cited.
Received: 26 August 2009

Accepted: 29 December 2009

\begin{abstract}
Background: Large regions of central and eastern Europe are recognized as areas where tickborne encephalitis virus (TBEV) is endemic, including countries neighbouring Denmark. It is therefore timely and relevant to determine if TBEV infections occur in Denmark. This study investigates the presence of antibodies against TBEV in a cross-section of the Danish canine population to assess the level of exposure to TBEV and possibly identify TBEV microfoci in Denmark.
\end{abstract}

Methods: Blood samples were collected from 125 dogs originating from five regions of Denmark between November 2005 and March 2006. Serum was tested by indirect ELISA. All positive and borderline samples were re-evaluated by neutralisation test (NT).

Results: The prevalence of TBEV serocomplex antibodies was $30 \%$ by ELISA and $4.8 \%$ by NT (with $100 \%$-neutralising capacity). The island of Bornholm was the only area in Denmark with NT positive samples.

Conclusions: The island of Bornholm is an area with a high risk of encountering TBEV microfoci. The presence of TBEV serocomplex antibodies in many sentinel animals from other parts of Denmark points toward existence of other TBEV microfoci. Discrepancies found between ELISA and NT results stress the importance of careful evaluation of serological tests, when interpreting results.

\section{Introduction}

Tick-borne encephalitis virus (TBEV), a flavivirus, is the cause of the most important arthropod-borne viral disease in central and eastern Europe. It is believed to result in at least 3000 human cases of tick-borne encephalitis annu- ally in Europe $[1,2]$. TBEV is transmitted to mammals, birds, reptiles and amphibians by ticks of the Ixodes family, predominantly by Ixodes ricinus $[3,4]$. The virus causes not only severe meningitis, meningoencephalitis and numerous deaths, but can also induce long-term debilitat- 
ing complications in patients that survive a severe form of the disease $[3,4]$. Canine TBE is characterized by lower morbidity, but a higher mortality rate, than human TBE, and dogs are often euthanized because of the severity of their clinical manifestations $[4,5]$. There is no cure for infection with TBEV and apart from the use of hyperimmunoglobulins in humans over the age of 14 [6], symptomatic therapy is the only means of providing patient support.

Viral existence and the maintenance of TBEV microfoci not only require a microhabitat favorable for Ixodes ticks, but suitable hosts and host population dynamics are also important $[7,8]$. Factors including habitat, seasonal variation and vector-host interactions contribute to the transmission of TBEV. Ixodes ricinus exist throughout Denmark and TBEV microfoci have been predicted in many parts of the country, which has raised concern about the establishment of TBEV in areas other than Bornholm [9]. Environmental change to warmer and more humid conditions encourages the spread of tick habitats and establishment of new TBEV microfoci, which pose the threat of new and more abundant infection centers [10].

In Denmark, TBE was first discovered in 1963 on Bornholm, an island of $588 \mathrm{~km}^{2}$ located in the Baltic Sea [11]. At the time when this study was performed, Bornholm was the only location in Denmark where TBEV microfoci had been documented $[12,13]$. TBEV serocomplex antibodies had, however, been detected in Danish wildlife, indicating that TBE transmission occurred in other areas than Bornholm [14] and, during the summer of 2009, TBEV was found in Ixodes ricinus ticks in Northern Zealand [15].

The aim of this study was to examine Danish dogs for serological evidence of infection with TBEV and to estimate the prevalence of TBEV serocomplex antibodies in the animals tested. Furthermore, the study intended to identify the location of potential TBEV risk areas in Denmark as well as possible risk factors associated with a positive titer in dogs. Finally, the use of anti-TBEV enzymelinked immunosorbent assay (ELISA) in dogs was evaluated for sensitivity and specificity based on the results of the anti-TBEV neutralization test (NT).

\section{Methods}

\section{Study population and materials}

The investigation was designed as a cross-sectional study, where dogs were used as sentinel animals and screened for presence of antibodies against TBEV. The study population consisted of clinically healthy dogs. Animals were recruited from five veterinary clinics from different regions of Denmark (Figure 1). Only dogs over the age of 4 years, and weighing more than $15 \mathrm{~kg}$, were included because dogs of this age and size were more likely to have

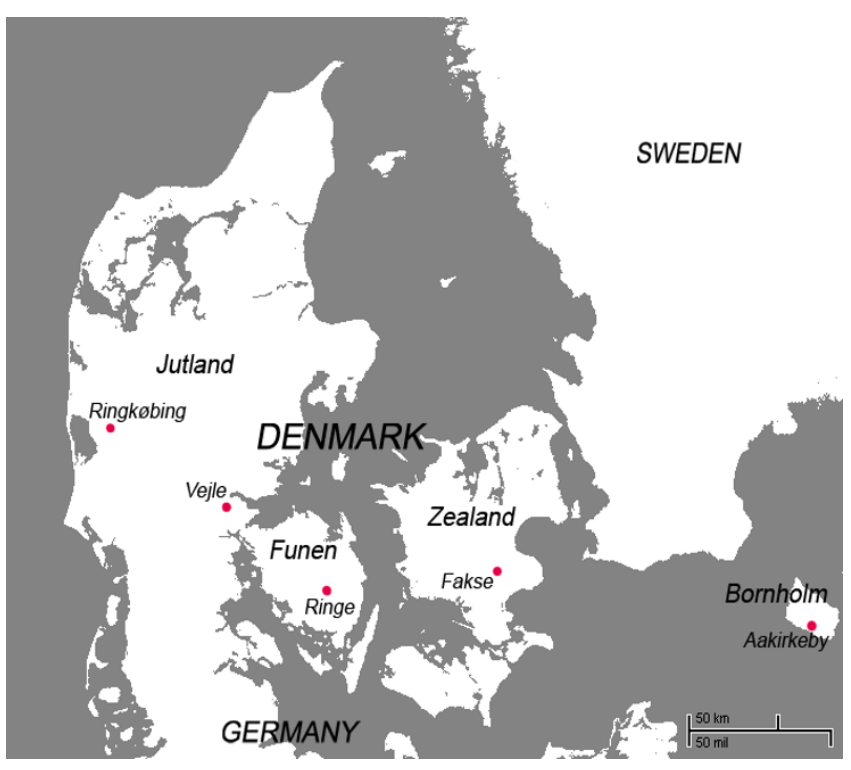

Figure I

Geographic distribution of the five veterinary clinics in Denmark that provided canine blood samples.

previously visited typical tick habitat such as fields or woodlands. Dogs that had previously travelled to TBE endemic areas outside of Denmark were excluded from the study. For each dog, the following data were collected: place of origin (owner's postal address), sampling month, age, breed, gender and degree of sample haemolysis.

Blood was collected in serum tubes and sent to the Central Laboratory, University of Copenhagen. The samples were centrifuged at $2560 \mathrm{~g}$ for two minutes (Heraeus Multifuge $1 \mathrm{~S}-\mathrm{R}$ ) and the serum was transferred to small vials, which were kept at $-18^{\circ} \mathrm{C}$ until the time of analysis.

Canine TBEV antibody positive blood samples were obtained from the University of Veterinary Medicine, Vienna, Austria and used as positive controls. Negative control samples were collected from young, small breed, urban-dwelling dogs from Copenhagen that had never travelled.

\section{Serology}

TBEV serocomplex antibodies were detected by a modified indirect ELISA. The ELISA kit, Enzygnost ${ }^{\varpi}$ Anti-TBE virus (IgG, IgM; Dade Behring, Deerfield, IL, USA; User's Manual March 2005 p.1-16) intended for use in humans was used in combination with anti-dog IgG conjugate (Bethyl Laboratories, Montgomery, TX, USA) at a dilution of 1:20,000. Canine serum samples were diluted to 1:40. To test the accuracy of the method and the micro titration plates, human negative and positive samples were included in each plate. Human control samples were diluted 1:20, as recommended by the manufacturer. All 
procedures were performed manually and antibody titer was determined by absorbance of samples in a spectrophotometer with a filter wavelength of $450 \mathrm{~nm}$ (Multiscan Ascent V1.24, Thermo Electron Corporation, Waltham, MA, USA). All samples were analysed in duplicate.

ELISA cut-off levels were determined using the manufacturer's recommendation and individual cut-off levels were set for each micro titration plate. Sample results were divided into three groups: negative, positive and borderline, with a cut-off value $\leq$ absorbance of sample $\leq$ cut-off +0.1 , as defined by the user's manual. Positive and borderline samples were sent to the Medical University of Vienna, Austria for double-testing by NT, carried out as described by Holzmann and colleagues [16]. Samples with $100 \%$ neutralising capacity, measured as $=1: 10$ in titer level, were considered NT positive. In addition, 5 randomly chosen negative samples were also sent to assess the sensitivity of the ELISA.

\section{Statistical analyses}

Association between selected potential risk factors such as location (postal address), sampling month, age, breed, gender and degree of sample haemolysis and TBEV antibody level was investigated using descriptive and statistical analyses (SAS ${ }^{\circledast} 9.1$ software and Fisher's exact test for statistical significance). The mean, median, standard deviation and variance of titer were determined by Proc Univariate. The extent of interaction and confounding was evaluated in a generalized linear model (Proc Mixed and Proc Genmod). NT results were analyzed using the frequency tables (Proc Freq). Finally, by comparing the results of the ELISA and NT, the specificity and sensitivity of the ELISA was assessed.

\section{Results}

A total of 125 canine blood samples were obtained. Of the 125 samples collected, 38 were positive for TBEV serocomplex antibodies and 19 were categorised as borderline. Figure 1 shows the veterinary clinics where samples were collected.

ELISA positive samples were found in all regions of Denmark included in the study, but a greater number of positive samples originated from Bornholm, where $50 \%$ of the ELISA samples were positive, compared to $34 \%, 30 \%$ and $21 \%$ on Zealand, Funen and Jutland. No statistically significant correlation was found between location (dog owner's postal address) and a positive titer $(P=0.1916$ by Fisher's exact test).

Analysis of all ELISA samples showed a seroprevalence of $30.4 \%$. Only six samples $(4.8 \%)$ were NT positive. Five of the ELISA and NT positive samples were from dogs living on Bornholm. The sixth dog originated from Zealand, but had previously travelled to Bornholm. The five seropositive samples from Bornholm correspond to a regional seroprevalence of $31 \%$. The three ELISA negative samples and two negative controls tested by NT were truly seronegative. The sensitivity of the ELISA was found to be $100 \%$ and the specificity $57.6 \%$ under the assumption that all 68 ELISA negative samples were negative.

The age of the dogs ranged from 4 to 15 years with an average of 7.5 years. The average age of ELISA positive dogs was 8.1 years. Although not statistically significant $(P=$ 0.0647 ), the risk of having developed antibodies against TBEV increased with the age of the dog (Figure 2).

Gender had a statistically significant correlation with titer $(P=0.0014$ by Fisher's exact test). Sixty per cent $(39 / 65)$ of the female samples were positive or borderline compared to $29 \%$ (17/59) of the male samples. One sample of unknown gender was omitted from the statistics. Dog breed and level of sample haemolysis was not statistically correlated with titer. No interaction or confounding bias was indicated between any of the investigated influencing factors (age, month, place of origin, gender). Using the NT data, the only significant influencing factor was place of origin i.e. the owner's postal address $(P<0.001$ Fisher's exact test).

\section{Discussion}

Our study assessed the prevalence of antibodies against TBEV in a cross-section of Danish dogs to be $4.8 \%$ by NT. By means of a modified, indirect ELISA, a seroprevalence of TBEV antibodies was found in $30.4 \%$ of the dogs. This prevalence is higher than that found in dogs by a similar method in Norway (16.4\%) [17] and that found in humans in TBEV endemic areas in Sweden (12\%) [18].

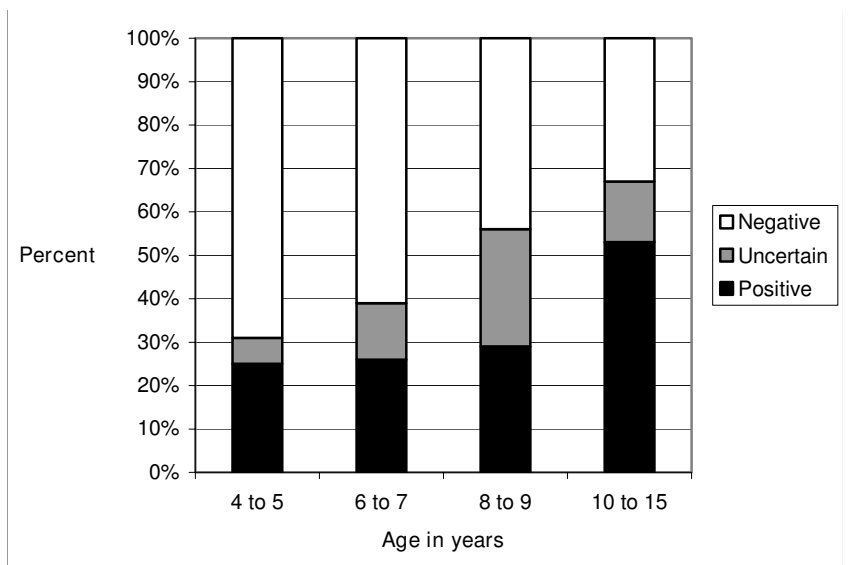

Figure 2

Distribution of tick-borne encephalitis virus ELISA positive, uncertain and negative canine serum samples grouped according to dog age. 
The seroprevalence found in the present study may be higher than that found in Norway because of a more widespread TBEV infection of ticks or because of differences in study population, i.e. greater number of urban dogs in the Norwegian study, or resulting from the possible presence of cross-reactive viruses in Denmark. Seroprevalences of $30-32 \%$ have been observed among roe deer (Capreolus capreolus) and forest workers on Bornholm [12,13,19]. Similar to this, we found a seroprevalence of $31 \%$ in dogs originating from Bornholm.

As argued in other studies of tick-borne diseases, the use of sentinel animals to indicate presence of an infectious agent is rewarding $[14,17,20,21]$. Dogs are good hosts for ticks because they move through undergrowth and tall grass at the right height for tick nymph and adult attachment. Our findings of antibodies against TBEV in Danish dogs indicate a risk for humans when passing through the same TBEV-infected tick habitats that lead to the infection in dogs.

Although the ELISA titer outcome was not statistically significant, the number of positive samples increased with increasing dog age. Antibodies are known to remain in circulation for extended periods of time and can therefore accumulate, resulting in the highest titers in elderly individuals $[22,23]$. The average age of positive dogs found by ELISA was 8.1 years, which is consistent with a similar study in Norway [17].

Our study showed a considerable discrepancy between ELISA and NT results. The NT revealed a prevalence of $100 \%$ neutralising antibodies in $4.8 \%$ of the dogs. This is much lower than that found by ELISA. Although ELISA is known as the method of choice for the detection of TBEV specific antibodies in serum [24], our study shows that use of ELISA as the sole serologic diagnostic method when testing dogs may be insufficient. The sensitivity of the ELISA kit, when used in humans, was reported to be $99.5 \%$ and the specificity, to $96.8 \%$ [17]. A recent evaluation of the ELISA kit in humans [25] showed sensitivity at $84 \%$ and specificity of $78 \%$. In light of the recent discovery of TBEV infected ticks and associated human cases (not evaluated by NT) on the island of Zealand, which had high ELISA anti-TBEV antibody titers, it is reasonable to speculate that these are real TBEV infections that have not lead to the production of neutralizing antibodies detectable by the methods used in this study. The occurrence of ELISA-positive dog sera and lack of NT positive samples from Zealand might be caused by changes in antigen profile during serial passage in the tick population (transovarial transmission [26]), which are reactivated by passage through mammalian hosts. However, at this time we can only speculate about the cause and note that high ELISA titers may have preceded the finding of human cases. Finally, studies on the prevalence of TBEV in Denmark are consistent with the emergence, disappearance and reoccurrence of TBEV microfoci, as has been reported from other parts of Europe in recent years $[10,27]$.

\section{Conclusion}

This study confirms that the island of Bornholm is a TBEV risk area in Denmark. Furthermore, our results suggest that the existence of TBEV microfoci in other parts of Denmark is likely. The study also emphasizes the need for careful evaluation of serological tests when interpreting results in the clinic.

\section{Abbreviations}

TBEV: Tick-borne encephalitis virus; ELISA: Enzymelinked immunosorbent assay; NT: Neutralisation test

\section{Competing interests}

Baxter Healthcare Corporation has partly financed the data collection but it has had no influence in the design, collection of results, interpretation or preparation of the manuscript at any time. The authors declare that they have no competing interests.

\section{Authors' contributions}

KESL and DSM participated in conceiving the study design, they carried out the ELISA testing and statistical analyses, created the figures and drafted the manuscript. KESL prepared the final manuscript. PMJ supported design of the study, provided technical assistance with the ELISA testing, helped with statistical analysis, and contributed to the manuscript. $\mathrm{GH}$ and $\mathrm{MB}$ contributed to the study design, participated in coordination and supervision of the project and helped to draft the manuscript. All authors have read and approved the final manuscript.

\section{Acknowledgements}

We thank the Baxter Healthcare Corporation for financial support. We are grateful to Michael Leschnik, DVM, The Clinic for Internal Medicine and Infectious Diseases, University of Veterinary Medicine, Vienna, Austria for his time and for providing positive canine controls. Finally, we thank the veterinarians from Ringe, Vejle, Ringkøbing, Aakirkeby and Fakse Veterinary clinics and the Copenhagen Veterinary Hospital for providing canine blood samples.

\section{References}

I. Gritsun TS, Lashkevich VA, Gould EA: Tick-borne encephalitis. Antiviral Res 2003, 57:129-46.

2. Bender A, Schulte-Altedorneburg G, Walther EU, Pfister HW: Severe tick borne encephalitis with simultaneous brain stem, bithalamic, and spinal cord involvement documented by MRI. J Neurol Neurosurg Psychiatry 2005, 76: I35-7.

3. Farkas R: Tick-borne Viral Encephalitis of Dogs and Cats. Guide to Major Vector-borne Diseases of Pets 2002:179-84.

4. Weissenbock H, Suchy A, Holzmann H: Tick-borne encephalitis in dogs: neuropathological findings and distribution of antigen. Acta Neuropathol 1998, 95:36 I-366.

5. Tipold A, Fatzer R, Holzmann H: Central European tick-born encephalitis in dogs [in German]. Kleintierpraxis 1993, 38:619-628. 
6. Arras C, Fescharek R, Gregersen JP: Do specific hyperimmunoglobulins aggravate clinical course of tick-borne encephalitis? Lancet 1996, 347:| 331 .

7. Freundt EA: The Western boundary of endemic tick-borne meningo-encephalitis in southern Scandinavia. Acta Path 1963, 57:87-103.

8. Jensen PM, Skarphedinsson S, Semenov A: Densities of the tick (Ixodes ricinus) and coexistence of the louping ill virus and tick borne encephalitis on the island of Bornholm [in Danish]. Ugeskr Læger 2004, 166:2563-2565.

9. Kristiansen K: TBE in Denmark - in particular on Bornholm. Int J Med Microbiol 2002, 29 I (Supl 33):62-63.

10. Sonenshine DE: Tick-borne Arboviral Diseases, Chapter 27. In Biology of Ticks 2nd edition. Oxford University Press, New York; 1993:157-16I.

11. Nuttall PA, Labuda M: Tick-Borne Encephalitis Subgroup. In Ecological Dynamics of Tick-Borne Zoonoses Edited by: Sonenshine DE, Mather TN. Oxford University Press, New York; 1994:35I-91.

12. Randolph SE: The shifting landscape of tick-borne zoonoses: tick-borne encephalitis and lyme borreliosis in Europe. Philos Trans R Soc Lond B Biol Sci 200I, 356: 1045-56.

13. Lindgren $E$, Talleklint L, Polfeldt T: Impact of climatic change on the northern latitude limit and population density of the disease-transmitting European tick Ixodes ricinus. Environ Health Perspect 2000, 108: I 19-23.

14. Skarphedinsson S, Jensen PM, Kristiansen K: Survey of tickborne infections in Denmark. Emerg Infect Dis 2005, I I:I055-6I.

15. Fomsgaard A, Christiansen CB, Bødker R: First identification of tick-borne encephalitis in Denmark outside of Bornholm August 2009. Eurosurveillance 2009, 14(36):

16. Holzmann $\mathrm{H}$, Kundi M, Stiasny K, Clement J, McKenna P, Kunz CH, Heinz FX: Correlation between elisa, hemagglutination inhibition and neutralizationstests after vaccination against tickborne encephalitis. I Med Virol 1996, 48: 102-107.

17. Csango PA, Blakstad E, Kirtz GC, Pedersen JE, Czettel B: Tick-borne encephalitis in southern Norway. Emerg Infect Dis 2004, 10:533-534.

18. Gustafson R, Svenungsson B, Gardulf A, Stiernstedt G, Forsgren M: Prevalence of tick-borne encephalitis and Lyme borreliosis in a defined Swedish population. Scand J Infect Dis 1990, 22:297-306.

19. The Danish Serum Institute: Tick-borne encephalitis on Bornholm [in Danish]. EPI nyt 17 200I [http://www.serum.dk/ sw1934.asp].

20. Takeda T, Ito T, Osada M, Takahashi K, Takashima I: Isolation of tick-borne encephalitis virus from wild rodents and a seroepizootiologic survey in Hokkaido, Japan. Am J Trop Med Hyg 1999, 60(2):287-291.

21. Rieger MA, Nübling M, Müller W, Hasselhorn H, Hofmann F: Foxes as indicators for TBE endemicity - a comparative serological investigation. Zentbl Bakteriol 1999, 289:610-618.

22. Kaiser $\mathrm{R}$ : The clinical and epidemiological profile of tickborne encephalitis in southern Germany 1994-98: a prospective study of 656 patients. Brain 1999, 122:2067-2078.

23. Leschnik MW, Kirtz GC, Thalhammer JG: Tick-borne encephalitis (TBE) in dogs. Int J Med Microbiol 2002, 291 (Suppl 33):66-69.

24. Holzmann H: Pitfalls in modern TBE sero-diagnosis (Extended summary). Zentbl Bakteriol 1999, 289:548-549.

25. Niedrig M, Vaisviliene D, Teichmann A, Klockmann U, Biel SS: Comparison of six different commercial IgG-ELISA kits for the detection of TBEV-antibodies. J Clin Virol 200।, 20: I79-182.

26. Labuda M, Jiang WR, Kaluzova M, Kožuch O, Nuttall PA, Weismann $P$, Elìckovia $E$, Zuffová $E$, Gould EA: Change in phenotype of tickborne encephalitis virus following passage in Ixodes ricinus ticks and associated amino acid substitution in the envelope protein. Virus Research 3 I(3):305-315.

27. Süss J, Schrader C, Abel U, Voigt W, Schosser R: Annual and seasonal variation of TBEV prevalence in ticks in selected hot spot areas in Germany using an RT-PCR: Results from 1997 and 1998. Zentbl Bakteriol 1999, 289:564-578.

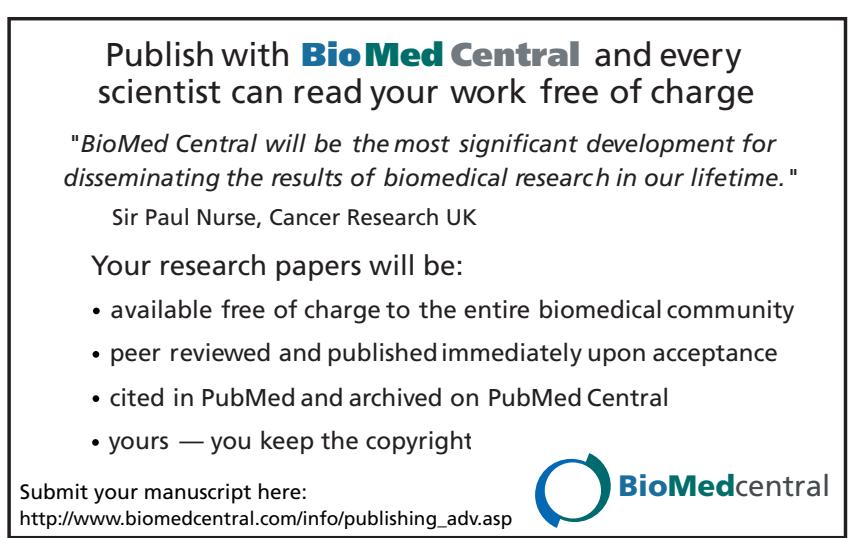

